\title{
EXISTENCE OF SOLUTIONS OF NONLINEAR INTEGRODIFFERENTIAL EQUATION WITH NONLOCAL CONDITION
}

\author{
K. BALACHANDRAN and M. CHANDRASEKARAN \\ Bharathiar University, Department of Mathematics \\ Coimbatore-641 046, Tamil Nadu, India
}

(Received February, 1996; Revised January, 1997)

\begin{abstract}
In this paper we prove the existence and uniqueness of local and global solutions of a nonlocal Cauchy problem for a class of integrodifferential equation. The method of semigroups and the contraction mapping principle are used to establish the results.

Key words: Existence of Solutions, Integrodifferential Equation, Semigroup Theory.
\end{abstract}

AMS subject classifications: $34 \mathrm{~K} 05,34 \mathrm{~K} 30$.

\section{Introduction}

The problem of existence of solutions of evolution equation with nonlocal conditions in Banach space was first studied by Byszewski [5]. In that paper he established the existence and uniqueness of mild, strong, and classical solutions of the following nonlocal Cauchy problem:

$$
\begin{gathered}
\frac{d u(t)}{d t}+A u(t)=f(t, u(t)), \quad t \in\left(t_{0}, t_{0}+a\right] \\
u\left(t_{0}\right)+g\left(t_{1}, t_{2}, \ldots, t_{p}, u(\cdot)\right)=u_{0}
\end{gathered}
$$

where $-A$ is the infinitesimal generator of a $C_{0}$-semigroup $T(t), t \geq 0$, on a Banach space $X, 0 \leq t_{0}<t_{1}<t_{2}<\ldots<t_{p} \leq t_{0}+a, a>0$, and $u_{0} \in X$ and $f:\left[t_{0}, t_{0}+a\right] \times$ $X \rightarrow X, g\left(t_{1}, \ldots, t_{p}, \cdot\right): X \rightarrow X$ are given functions. Subsequently, Byszewski [2-4, 6-8] investigated the same type of problem stated to a different class of evolution equations in Banach space.

The purpose of this paper is to prove the existence and uniqueness of local solution for an integrodifferential equation with nonlocal conditions of the form:

$$
\frac{d u(t)}{d t}+A u(t)=f(t, u(t))+\int_{0}^{t} h\left(t, s, u(s), \int_{0}^{s} k(s, \tau, u(\tau)) d \tau\right) d s
$$




$$
u(0)+g\left(t_{1}, t_{2}, \ldots, t_{p}, u(\cdot)\right)=u_{0} .
$$

Here we assume that $-A$ is the infinitesimal generator of a bounded analytic semigroup of linear operator $X(t), t \geq 0$, in a Banach space $Z$. The operator $A^{\alpha}$ can be defined for $0 \leq \alpha<1$, and $A^{\alpha}$ is a closed linear invertible operator with domain $D\left(A^{\alpha}\right)$ dense in $Z$. The closedness of $A^{\alpha}$ implies that $D\left(A^{\alpha}\right)$, endowed with the graph norm of $A^{\alpha},\|\| Z\|\|=\|Z\|+\left\|A^{\alpha} z\right\|$, is a Banach space. Since $A^{\alpha}$ is invertible, its graph norm \|\|$\cdot\|\|$ is equivalent to the norm $\|Z\|_{\alpha}=\left\|A^{\alpha} Z\right\|$. Thus, $D\left(A^{\alpha}\right)$ equipped with the norm $\|\cdot\|_{\alpha}$, is a Banach space, which we denote by $Z_{\alpha}$. From this definition, it is clear that $0<\alpha<\beta$ implies $Z_{\alpha} \supset Z_{\beta}$ and that the embedding of $Z_{\beta}$ in $Z_{\alpha}$ is continuous. Take $J=[0, a]$. Let $f: J \times Z_{\alpha} \rightarrow Z, h: J \times J \times$ $Z_{\alpha} \times Z_{\alpha} \rightarrow Z, \quad k: J \times J \times Z \rightarrow Z_{\alpha}, \quad$ and $g\left(t_{1}, \ldots, t_{p}, \cdot\right): Z_{\alpha} \rightarrow Z$ be given nonlinear operators. The notation $g\left(t_{1}, \ldots, t_{p}, u(\cdot)\right)$ is used in the sense that in the place of '.' we can substitute only an element of the set $\left\{t_{1}, \ldots, t_{p}\right\}$.

The results obtained in this paper generalize the Theorem 6.3.1 of Pazy [10] about the Cauchy problem. As in $[1-3,6,7,9]$, the nonlocal condition (2) in this paper can be used in physics with a better effect than the classical condition $u(0)=u_{0}$, since condition (2) is usually more suitable for physical measurements than the classical one.

\section{Existence Theorems}

Theorem 2.1: Assume that

(i) $\quad-A$ is the infinitesimal generator of a bounded analytic semigroup of linear operator $X(t), t>0$, in $Z$.

(ii) For $0 \leq \alpha<1$, the fractional power $A^{\alpha}$ satisfies $\left\|A^{\alpha} X(t)\right\| \leq C_{\alpha} t^{-\alpha}$ for $t>0$, where $C_{\alpha}$ is a real constant.

(iii) $0 \in \rho(-A)$, the resolvent set.

(iv) For an open subset $D$ of $J \times Z_{\alpha}, f: D \rightarrow Z$ satisfies the condition, if for every $(t, u) \in D$ there is a neighborhood $V \subset D$ and constants $L \geq 0$, $0<\Theta \leq 1$, such that

$$
\left\|f\left(t_{1}, u_{1}\right)-f\left(t_{2}, u_{2}\right)\right\| \leq L\left(\left|t_{1}-t_{2}\right|^{\Theta}+\left\|u_{1}-u_{2}\right\|_{\alpha}\right)
$$

for all $\left(t_{i}, u_{i}\right) \in V, i=1,2$.

(v) For an open subset $E$ of $J \times J \times Z_{\alpha} \times Z_{\alpha}, h: E \rightarrow Z$ satisfies the condition, if for every $(t, s, u, v) \in E$ there is a neighborhood $U \subset E$ and constants $L_{1} \geq 0,0<\Theta \leq 1$, such that

$$
\begin{gathered}
\left\|h\left(t_{1}, s_{1}, u_{1}, v_{1}\right)-h\left(t_{2}, s_{2}, u_{2}, v_{2}\right)\right\| \\
\left.\leq L_{1}\left(\left|t_{1}-t_{2}\right|^{\theta}+\left|s_{1}-s_{2}\right|^{\theta}\right)+\left\|u_{1}-u_{2}\right\|_{\alpha}+\left\|v_{1}-V_{2}\right\|_{\alpha}\right)
\end{gathered}
$$

for all $\left(t_{i}, s_{i}, u_{i}, v_{i}\right) \in U, i=1,2$.

(vi) For an open subset $P$ of $J \times J \times Z, k: P \rightarrow Z$ satisfies the condition, if for every $(t, s, u) \in P$ there is a neighborhood $W \subset P$ and constants $L_{2} \geq 0$,

$0<\Theta \leq 1$, such that 


$$
\begin{gathered}
\left\|k\left(t_{1}, s_{1}, u_{1}\right)-k\left(t_{2}, s_{2}, u_{2}\right)\right\| \\
\leq L_{2}\left(\left|t_{1}-t_{2}\right|^{\theta}+\left|s_{1}-s_{2}\right|^{\theta}+\left\|u_{1}-u_{2}\right\|_{\alpha}\right)
\end{gathered}
$$

for all $\left(t_{i}, s_{i}, u_{i}\right) \in W, i=1,2$.

(vii) $g: J^{p} \times Z_{\alpha} \rightarrow Z$ and there exists constants $B^{*}>0$ and $L^{*}>0$ such that

$$
\left\|A^{\alpha} g\left(t_{1}, \ldots, t_{p}, u(\cdot)\right)\right\| \leq B^{*} \text { for } 0 \leq t<a
$$

and

$$
\left\|g\left(t_{1}, \ldots, t_{p}, u_{1}(\cdot)\right)-g\left(t_{1}, \ldots, t_{p}, u_{2}(\cdot)\right)\right\| \leq L^{*}\left\|u_{1}-u_{2}\right\|_{\alpha} .
$$

Then the nonlocal Cauchy problem (3), (4) has a unique local solution $u \in C([0, a)$ : $Z) \cap C^{1}((0, a): Z)$.

Proof: Choose $t^{*}>0$ and $\delta>0$ such that estimates (5), (6), and (7) hold on the sets

$V=\left\{(t, u): 0 \leq t \leq t^{*},\left\|u-u_{0}\right\| \leq \delta\right\}$,

$U=\left\{(t, s, u, v): 0 \leq t, s \leq t^{*},\left\|u-u_{0}\right\| \leq \delta,\left\|v-v_{0}\right\| \leq \delta\right\}$, and

$W=\left\{(t, s, u): 0 \leq t, s \leq t^{*},\left\|u-u_{0}\right\| \leq \delta\right\}$, respectively.

Let $B=\max _{0}\left\|f\left(t, \overline{u_{0}}\right)\right\|$ and

$$
H=\max _{0 \leq t, s \leq t^{*}}\left\|h\left(t, s, u_{0}, \int_{0}^{s} k\left(s, \tau, u_{0}\right) d \tau\right)\right\|
$$

and choose $a$ such that for $0 \leq t<a$,

$$
\left\|X(t) A^{\alpha} u_{0}-A^{\alpha} u_{0}\right\|<\delta / 4
$$

and

$$
\left\|X(t) A^{\alpha} g\left(t_{1}, \ldots, t_{p}, u(\cdot)\right)-A^{\alpha} g\left(t_{1}, \ldots, t_{p}, u(\cdot)\right)\right\|<\delta / 4
$$

$$
\begin{gathered}
0<a<\min \left\{t^{*},\left[\delta / 2(1-\alpha) C_{\alpha}^{-1}\left(L \delta+B+L B^{*}+L_{1} \delta a+L_{1} B^{*} a\right.\right.\right. \\
\left.\left.\left.+L_{1} L_{2} \delta a^{2}+L_{1} L_{2} B^{*} a^{2}+H a\right)^{-1}\right]^{(1 /(1-\alpha))}\right\} .
\end{gathered}
$$

Let $Y$ be the Banach space $C((0, a]: Z)$ with usual supremum norm which we denote by $\|\cdot\|_{Y}$. Define a map $F: Y \rightarrow Y$ by

$F y(t)$

$$
\begin{gathered}
=X(t) A^{\alpha} u_{0}-X(t) A^{\alpha} g\left(t_{1}, \ldots, t_{p}, A^{-\alpha} y(\cdot)\right)+\int_{0}^{t} A^{\alpha} X(t-s) f\left(s, A^{-\alpha} y(s)\right) d s \\
+\int_{0}^{t} A^{\alpha} X(t-s) \int_{0}^{s} h\left(s, \tau, A^{-\alpha} y(\tau), \int_{0}^{\tau} k\left(\tau, \mu, A^{-\alpha} y(\mu)\right) d \mu\right) d \tau d s .
\end{gathered}
$$

Obviously, $F y(0)=A^{\alpha} u_{0}-A^{\alpha} g$. Let $S$ be the nonempty closed and bounded subset of $Y$ defined by

$$
S=\left\{y: y \in Y, y(0)=A^{\alpha} u_{0}-A^{\alpha} g,\left\|y(t)-\left(A^{\alpha} u_{0}-A^{\alpha} g\right)\right\| \leq \delta\right\} .
$$


For $y \in S$, we have

$$
\begin{aligned}
& \left\|F y(t)-\left(A^{\alpha} u_{0}-A^{\alpha} g\right)\right\| \leq\left\|X(t) A^{\alpha} u_{0}-A^{\alpha} u_{0}\right\| \\
& +\left\|X(t) A^{\alpha} g\left(t_{1}, \ldots, t_{p}, A^{-\alpha} y(\cdot)\right)-A^{\alpha} g\left(t_{1}, \ldots, t_{p}, A^{-\alpha} y(\cdot)\right)\right\| \\
& +\int_{0}^{t}\left\|A^{\alpha} X(t-s)\left[f\left(s, A^{-\alpha} y(s)\right)-f\left(s, u_{0}\right)\right]\right\| d s+\int_{0}^{t}\left\|A^{\alpha} X(t-s) f\left(s, u_{0}\right)\right\| d s \\
& +\int_{0}^{t} \| A^{\alpha} X(t-s)\left[\int_{0}^{s} h\left(s, \tau, A^{-\alpha} y(\tau), \int_{0}^{\tau} k\left(\tau, \mu, A^{-\alpha} y(\mu)\right) d \mu\right) d \tau\right. \\
& \left.-\int_{0}^{s} h\left(s, \tau, u_{0}, \int_{0}^{\tau} k\left(\tau, \mu, u_{0}\right) d \mu\right) d \tau\right] \| d s \\
& +\int_{0}^{t}\left\|A^{\alpha} X(t-s) \int_{0}^{s} h\left(s, \tau, u_{0}, \int_{0}^{\tau} k\left(\tau, \mu, u_{0}\right) d \mu\right) d \tau\right\| d s \\
& \leq \delta / 4+\delta / 4+\int_{0}^{t}\left\|A^{\alpha} X(t-s) L\left[A^{-\alpha} y(s)-\left(u_{0}-g\right)-g\right]\right\| d s \\
& +B \int_{0}^{t}\left\|A^{\alpha} X(t-s)\right\| d s+\int_{0}^{t} \| A^{\alpha} X(t-s)\left\{L _ { 1 } \left[\left(A^{-\alpha} y(\tau)-u_{0}\right)\right.\right. \\
& \left.\left.+L_{2}\left(A^{-\alpha} y(\mu)-u_{0}\right) a\right] a\right\} \| d s+C_{\alpha} a^{(1-\alpha)}(1-\alpha)^{-1} H a \\
& \leq \delta / 2+C_{\alpha}\left(L \delta+B+L B^{*}\right) a^{(1-\alpha)}(1-\alpha)^{-1} \\
& +C_{\alpha} a^{(1-\alpha)}(1-\alpha)^{-1}\left\{L_{1}\left(\delta+B^{*}+L_{2}\left(\delta+B^{*}\right) a\right) a\right\}+C_{\alpha} a^{(1-\alpha)}(1-\alpha)^{-1} H a \\
& \leq \delta / 2+C_{\alpha} a^{(1-\alpha)}(1-\alpha)^{-1}\left\{L \delta+B+L B^{*}+L_{1} \delta a+L_{1} B^{*} a+L_{1} L_{2} \delta a^{2}\right. \\
& \left.+L_{1} L_{2} B^{*} a^{2}+H a\right\} \leq \delta / 2+\delta / 2=\delta .
\end{aligned}
$$

Therefore, $F$ maps $S$ into itself. Moreover, if $y_{1}, y_{2} \in S$, then

$$
\begin{aligned}
& \left\|F y_{1}(t)-F y_{2}(t)\right\| \\
& \leq\left\|X(t)\left(A^{\alpha} g\left(t_{1}, \ldots, t_{p}, A^{-\alpha} y_{1}(\cdot)\right)-A^{\alpha} g\left(t_{1}, \ldots, t_{p}, A^{-\alpha} y_{2}(\cdot)\right)\right)\right\| \\
& \quad+\int_{0}^{t}\left\|A^{\alpha} X(t-s)\left[f\left(s, A^{-\alpha} y_{1}(s)\right)-f\left(s, A^{-\alpha} y_{2}(s)\right)\right]\right\| d s
\end{aligned}
$$




$$
\begin{gathered}
+\int_{0}^{t} \| A^{\alpha} X(t-s)\left[\int_{0}^{s} h\left(s, \tau, A^{-\alpha} y_{1}(\tau), \int_{0}^{\tau} k\left(\tau, \mu, A^{-\alpha} y_{1}(\mu)\right) d \mu\right)\right. \\
\left.\quad-\int_{0}^{s} h\left(s, \tau, A^{-\alpha} y_{2}(\tau), \int_{0}^{\tau} k\left(\tau, \mu, A^{-\alpha} y_{2}(\mu)\right) d \mu\right) d \tau\right] \| d s \\
\leq C_{\alpha} a^{(1-\alpha)}(1-\alpha)^{-1} L^{*}\left\|y_{1}-y_{2}\right\|_{Y}+C_{\alpha} a^{(1-\alpha)}(1-\alpha)^{-1} L\left\|y_{1}-y_{2}\right\|_{Y} \\
+C_{\alpha} a^{(1-\alpha)}(1-\alpha)^{-1} L_{1}\left[\left(\left\|y_{1}-y_{2}\right\|_{Y}+L_{2}\left\|y_{1}-y_{2}\right\|_{Y} a\right) a\right] \\
\leq C_{\alpha} a^{(1-\alpha)}(1-\alpha)^{-1}\left[L^{*}+L+L_{1}\left(1+L_{2} a\right) a\right]\left\|y_{1}-y_{2}\right\|_{Y} \\
\leq(1 / 2)\left\|y_{1}-y_{2}\right\|_{Y},
\end{gathered}
$$

which implies that

$$
\left\|F y_{1}-F y_{2}\right\|_{Y} \leq(1 / 2)\left\|y_{1}-y_{2}\right\|_{Y}
$$

By the contraction mapping theorem, mapping $F$ has a unique fixed point $y \in S$. This fixed point satisfies the integral equation

$$
\begin{gathered}
y(t)=X(t) A^{\alpha} u_{0}-X(t) A^{\alpha} g\left(t_{1}, \ldots, t_{p}, A^{-\alpha} y(\cdot)\right) \\
+\int_{0}^{t} A^{\alpha} X(t-s) f\left(s, A^{-\alpha} y(s)\right) d s \\
+\int_{0}^{t} A^{\alpha} X(t-s) \int_{0}^{s} h\left(s, \tau, A^{-\alpha} y(\tau), \int_{0}^{\tau} k\left(\tau, \mu, A^{-\alpha} y(\mu)\right) d \mu\right) d \tau d s .
\end{gathered}
$$

From (5), (6) and the continuity of $y$ it follows that

$$
t \rightarrow f\left(t, A^{-\alpha} y(t)\right) \text { and } t \rightarrow h\left(t, s, A^{-\alpha} y(s), \int_{0}^{s} k\left(s, \tau, A^{-\alpha} y(\tau)\right) d \tau\right.
$$

are continuous on $[0, a]$, and, hence, there exist constants $N$ and $H^{*}$ such that

and

$$
\left\|f\left(t, A^{-\alpha} y(t)\right)\right\| \leq N
$$

$$
\| h\left(t, s, A^{-\alpha} y(s), \int_{0}^{s} k\left(s, \tau, A^{-\alpha} y(\tau)\right) d \tau \| \leq H^{*}\right.
$$

Note that for every $\beta$ satisfying $0<\beta<1-\alpha$ and every $0<h<1$, we have by Theorem 2.6.13 of [10] that

$$
\left\|(X(h)-I) A^{\alpha} X(t-s)\right\|
$$




$$
\leq C_{\beta} h^{\beta}\left\|A^{\alpha+\beta} X(t-s)\right\| \leq C h^{\beta}(t-s)^{-(\alpha-\beta)} \text { for some } C>0
$$

If $0<t<t+h \leq a$, then

$$
\begin{gathered}
\|y(t+h)-y(t)\| \leq\left\|(X(h)-I) A^{\alpha} X(t) u_{0}\right\| \\
+\left\|(X(h)-I) A^{\alpha} X(t) g\left(t_{1}, \ldots, t_{p}, A^{-\alpha} y(\cdot)\right)\right\| \\
+\int_{0}^{t}\left\|(X(h)-I) A^{\alpha} X(t-s) f\left(s, A^{-\alpha} y(s)\right)\right\| d s \\
+\int_{t}^{t+h}\left\|A^{\alpha} X(t+h-s) f\left(s, A^{-\alpha} y(s)\right)\right\| d s \\
+\int_{t}^{t}\left\|(X(h)-I) A^{\alpha} X(t-s) \int_{0}^{s} h\left(s, \tau, A^{-\alpha} y(\tau), \int_{0}^{\tau} k\left(\tau, \mu, A^{-\alpha} y(\mu)\right) d \mu\right) d \tau\right\| d s \\
\left\|A^{\alpha} X(t+h-s) \int_{0}^{s} h\left(s, \tau, A^{-\alpha} y(\tau), \int_{0}^{\tau} k\left(\tau, \mu, A^{-\alpha} y(\mu)\right) d \mu\right) d \tau\right\| d s \\
=I_{1}+I_{2}+I_{3}+I_{4}+I_{5}+I_{6}
\end{gathered}
$$

Using (vii), (11), (12), and (13) we find that

$$
\begin{gathered}
I_{1} \leq C t^{-(\alpha+\beta)} h^{\beta} \leq M_{1} h^{\beta} \\
I_{2} \leq C B^{*} t^{-(\alpha+\beta)} h^{\beta} \leq M_{2} h^{\beta} \\
I_{3} \leq C N h^{\beta} \int_{0}^{t}(t-s)^{-(\alpha+\beta)} d s \leq M_{3} h^{\beta} \\
I_{4} \leq N C_{\alpha} \int_{t}^{t+h}(t+h-s)^{-\alpha} d s=N C_{\alpha}(1-\alpha)^{-1} h^{(1-\alpha)} \leq M_{4} h^{\beta} \\
I_{5} \leq C h^{\beta} H^{*} a \int_{0}^{t}(t-s)^{-(\alpha+\beta)} d s \leq M_{5} h^{\beta} \\
I_{6} \leq H^{*} C_{\alpha} a \int_{t}^{t+h}(t+h-s)^{-\alpha} d s=H^{*} a C_{\alpha}(1-\alpha)^{-1} h^{(1-\alpha)} \leq M_{6} h^{\beta} .
\end{gathered}
$$

Here, $M_{1}$ and $M_{2}$ depend on $t$ and vanish at $t \rightarrow 0$, but $M_{3}, M_{4}, M_{5}$, and $M_{6}$ can be selected to be independent of $t \in J$. Combining (14) with these estimates it follows 
that for every $t^{\prime}>0$ there is a constant $C$ such that $\|y(t)-y(s)\| \leq C|t-s|^{\beta}$ for $0 \leq t^{\prime} \leq t, s \leq a$ and therefore, $y$ is locally Hölder continuous on $(0, a]$. The local Hölder continuity of $t \rightarrow f\left(t, A^{-\alpha} y(t)\right)$ follows from

$$
\begin{gathered}
\left\|f\left(t, A^{-\alpha} y(t)\right)-f\left(s, A^{-\alpha} y(s)\right)\right\| \leq L\left(|t-s|^{\Theta}+\|y(t)-y(s)\|\right) \\
\leq C_{1}\left(|t-s|^{\Theta}+|t-s|^{\beta}\right) \text { for some } C_{1}>0
\end{gathered}
$$

and the local Hölder continuity of

follows from

$$
t \rightarrow h\left(t, s, A^{-\alpha} y(s), \int_{0}^{s} k\left(s, \tau, A^{-\alpha} y(\tau) d \tau\right)\right.
$$

$$
\begin{gathered}
\| h\left(t, s, A^{-\alpha} y(s), \int_{0}^{s} k\left(s, \tau, A^{-\alpha} y(\tau)\right) d \tau\right) \\
-h\left(t, \mu, A^{-\alpha} y(\mu), \int_{0}^{s} k\left(\mu, \phi, A^{-\alpha} y(\phi)\right) d \phi\right) \| \\
\leq L_{1}\left\{|s-\mu|^{\Theta}+\|y(s)-y(\mu)\|+L_{2}\left(|s-\mu|^{\Theta}\right.\right. \\
\left.\left.\quad+|\tau-\phi|^{\Theta}+\|y(\tau)-y(\phi)\|\right) a\right\} \\
\leq L_{1}\left\{|s-\mu|^{\Theta}+|s-\mu|^{\beta}+L_{3}\left(|s-\mu|^{\Theta}+|\tau-\phi|^{\Theta}+|\tau-\phi|^{\beta}\right) a\right\}
\end{gathered}
$$

for some $L_{3}>0$. Let $y$ be a solution of (10). Consider the inhomogeneous initial value problem

$$
\begin{gathered}
\frac{d u(t)}{d t}+A u(t)=f\left(t, A^{-\alpha} y(t)\right) \\
+\int_{0}^{t} h\left(t, s, A^{-\alpha} y(s), \int_{0}^{s} k\left(s, \tau, A^{-\alpha} y(\tau)\right) d \tau\right) d s \\
u(0)+g\left(t_{1}, \ldots, t_{p}, A^{-\alpha} y(\cdot)\right)=u_{0} .
\end{gathered}
$$

This problem has a unique solution $u \in C^{1}((0, a]: Z)[10]$, which is given by

$$
\begin{aligned}
u(t) & =X(t) u_{0}-X(t) g\left(t_{1}, \ldots, t_{p}, A^{-\alpha} y(\cdot)\right)+\int_{0}^{t} X(t-s) f\left(s, A^{-\alpha} y(s)\right) d s \\
& +\int_{0}^{t} X(t-s) \int_{0}^{s} h\left(s, \tau, A^{-\alpha} y(\tau), \int_{0}^{\tau} k\left(\tau, \mu, A^{-\alpha} y(\mu)\right) d \mu\right) d \tau d s .
\end{aligned}
$$

For $t>0$, each term of (17) is in $D(A)$ and a fortiori in $D\left(A^{\alpha}\right)$. Operating on both sides of (17) with $A^{\alpha}$ we find that 


$$
\begin{gathered}
A^{\alpha} u(t)=X(t) A^{\alpha} u_{0}-X(t) A^{\alpha} g\left(t_{1}, \ldots, t_{p}, A^{-\alpha} y(\cdot)\right) \\
+\int_{0}^{t} A^{\alpha} X(t-s) f\left(s, A^{-\alpha} y(s)\right) d s \\
+\int_{0}^{t} A^{\alpha} X(t-s) \int_{0}^{s} h\left(s, \tau, A^{-\alpha} y(\tau), \int_{0}^{\tau} k\left(\tau, \mu, A^{-\alpha} y(\mu) d \mu\right) d \tau d s .\right.
\end{gathered}
$$

From (10) the right-hand side of (18) equals $y(t)$ and therefore $u(t)=A^{-\alpha} y(t)$ and by (17), $u$ is a $C^{1}((0, a]: Z)$ solution of $(3),(4)$. The uniqueness of $u$ follows from the uniqueness of the solutions of $(10)$ and $(15),(16)$. Hence, the theorem is proved.

Next, we shall prove the existence of global solutions of (3), (4).

Theorem 2.2: Let $0 \in \rho(-A)$ and let $-A$ be the infinitesimal generator of an analytic semigroup $X(t)$ satisfying $\|X(t)\| \leq M$ for $t \geq 0$. Let $f: I \times Z_{\alpha} \rightarrow Z, h: I \times$ $I \times Z_{\alpha} \times Z_{\alpha} \rightarrow Z$, and $g\left(t_{1}, \ldots, t_{p}, u(\cdot)\right): I^{p} \times Z_{\alpha} \rightarrow Z_{\alpha}$ satisfy (5), (6), and (7), respectively, with $I=[0, \infty)$. If there are continuous nondecreasing real valued functions $q_{1}(t)$ and $q_{2}(t)$ such that

and

$$
\|f(t, u)\| \leq q_{1}(t)\left(1+\|u\|_{\alpha}\right)
$$

$$
\left\|h\left(t, s, u, \int_{0}^{s} k(s, \tau, u) d \tau\right)\right\| \leq q_{2}(t)\left(1+\|u\|_{\alpha}\right)
$$

for $t \geq 0, u \in Z_{\alpha}$, then for every $u_{0} \in Z_{\alpha}$, equations (3), (4) have a unique solution $u$.

Proof: As in the proof of Theorem 2.1, the solution of (3) can be continued as long as $\|u(t)\|_{\alpha}$ remains bounded. It is enough to prove that if $u$ exists on $[0, a)$ then $\|u(t)\|_{\alpha}$ is bounded as $t \rightarrow a$.

Since

$$
\begin{aligned}
A^{\alpha} u(t)= & X(t) A^{\alpha} u_{0}-X(t) A^{\alpha} g\left(t_{1}, \ldots, t_{p}, u(\cdot)\right)+\int_{0}^{t} A^{\alpha} X(t-s) f(s, u(s)) d s \\
& +\int_{0}^{t} A^{\alpha} X(t-s) \int_{0}^{s} h\left(s, \tau, u(\tau), \int_{0}^{\tau} k(\tau, \mu, u(\mu)) d \mu\right) d \tau d s,
\end{aligned}
$$

then

$$
\begin{gathered}
\|u(t)\|_{\alpha} \leq M\left\|A^{\alpha} u_{0}\right\|+M\left\|A^{\alpha} g\left(t_{1}, \ldots, t_{p}, u(\cdot)\right)\right\| \\
+\int_{0}^{t}\left\|A^{\alpha} X(t-s) f(s, u(s))\right\| d s \\
+\int_{0}^{t}\left\|A^{\alpha} X(t-s) \int_{0}^{s} h\left(s, \tau, u(\tau), \int_{0}^{\tau} k(\tau, \mu, u(\mu)) d \mu\right) d \tau\right\| d s \\
\leq M\left[\left\|A^{\alpha} u_{0}\right\|+\left\|A^{\alpha} g\left(t_{1}, \ldots, t_{p}, u(\cdot)\right)\right\|\right]
\end{gathered}
$$




$$
\begin{aligned}
& +q_{1}(t) a^{(1-\alpha)}(1-\alpha)^{-1}+q_{1}(t) \int_{0}^{t}(t-s)^{-\alpha}\|u(s)\|_{\alpha} d s \\
& +q_{2}(t) a^{(1-\alpha)}(1-\alpha)^{-1}+q_{2}(t) \int_{0}^{t}(t-s)^{-\alpha}\|u(s)\|_{\alpha} d s \\
& \leq M\left[\left\|A^{\alpha} u_{0}\right\|+\left\|A^{\alpha} g\left(t_{1}, \ldots, t_{p}, u(\cdot)\right)\right\|\right] \\
& +q_{1}(t) a^{(1-\alpha)}(1-\alpha)^{-1}+q_{2}(t) a^{(1-\alpha)}(1-\alpha)^{-1} \\
& +\left(q_{1}(t)+q_{2}(t)\right) \int_{0}^{t}(t-s)^{-\alpha}\|u(s)\|_{\alpha} d s
\end{aligned}
$$

By Gronwall's inequality, we get

$$
\|u(t)\|_{\alpha} \leq C \text { on }[0, a) .
$$

Hence, the proof.

\section{Acknowledgement}

The authors are thankful to Professor J. Dshalalow for his kind help.

\section{References}

[1] Balachandran, K. and Ilamaran, S., Existence and uniqueness of mild and strong solutions of a semilinear evolution equation with nonlocal conditions, Indian J. Pure Appl. Math. 25 (1994), 411-418.

[2] Byszewski, L., Strong maximum principles for parabolic nonlinear problems with nonlocal inequalities together with integrals, J. App. Math. Stoch. Anal. 3 (1990), 65-79.

[3] Byszewski, L., Existence and uniqueness of solutions of nonlocal problems for hyperbolic equation $u_{x t}=F\left(x, t, u, u_{x}\right)$, J. Math. Stoch. Anal. 3 (1990), 163-168.

[4] Byszewski, L., Theorem about existence and uniqueness of continuous solution of nonlocal problem for nonlinear hyperbolic equation, Appl. Anal. 40 (1991), 173-180.

[5] Byszewski, L., Theorems about the existence and uniqueness of solutions of a semilinear evolution nonlocal Cauchy problem, J. Math. Anal. Appl. 162 (1991), 494-506.

[6] Byszewski, L., Strong maximum principles for parabolic nonlinear problems with nonlocal inequalities together with arbitrary functions, J. Math. Anal. Appl. 156 (1991), 457-470.

[7] Byszewski, L., Existence of approximate solution to abstract nonlocal Cauchy problem, J. Appl. Math. Stoch. Anal. 5 (1992), 363-374.

[8] Byszewski, L., Uniqueness criterion for solution of abstract nonlocal Cauchy 
problem, J. Appl. Math. Stoch. Anal. 6 (1993), 49-54.

[9] Jackson, D., Existence and uniqueness of solutions to semilinear nonlocal parabolic equations, J. Math. Anal. Appl. 172 (1993), 256-265.

[10] Pazy, A., Semigroups of Linear Operators and Applications to Partial Differential Equations, Springer-Verlag, New York 1983. 


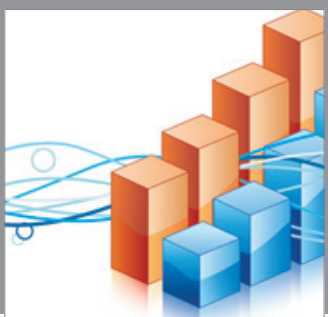

Advances in

Operations Research

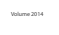

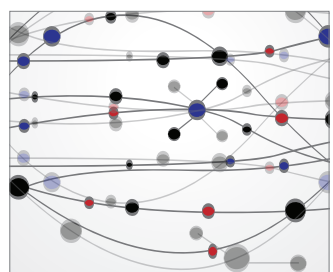

\section{The Scientific} World Journal
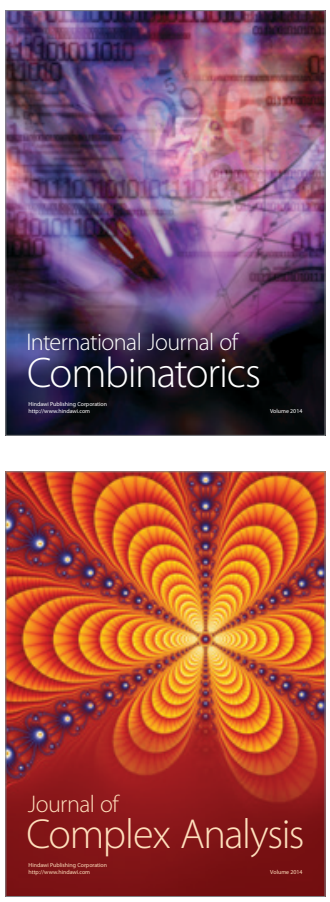

International Journal of

Mathematics and

Mathematical

Sciences
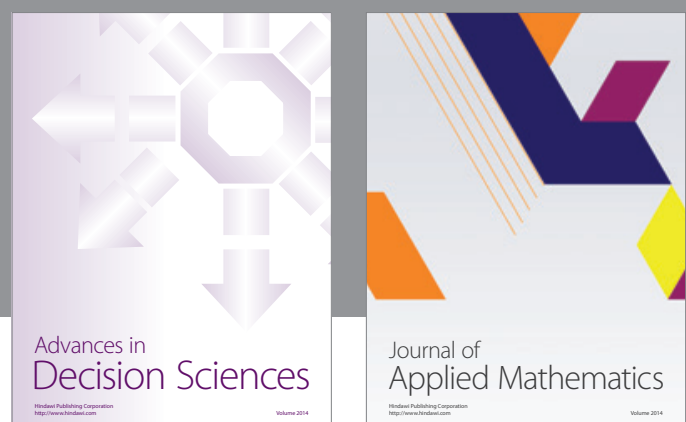

Journal of

Applied Mathematics
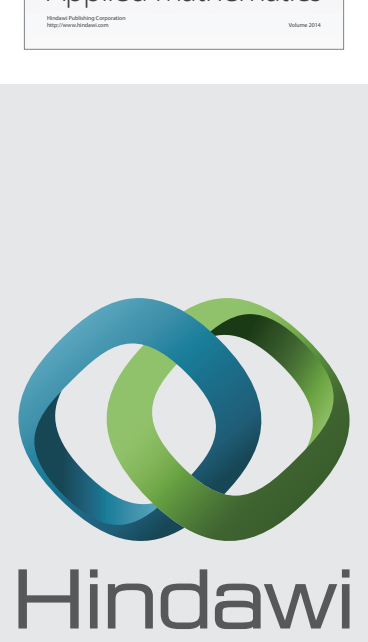

Submit your manuscripts at http://www.hindawi.com
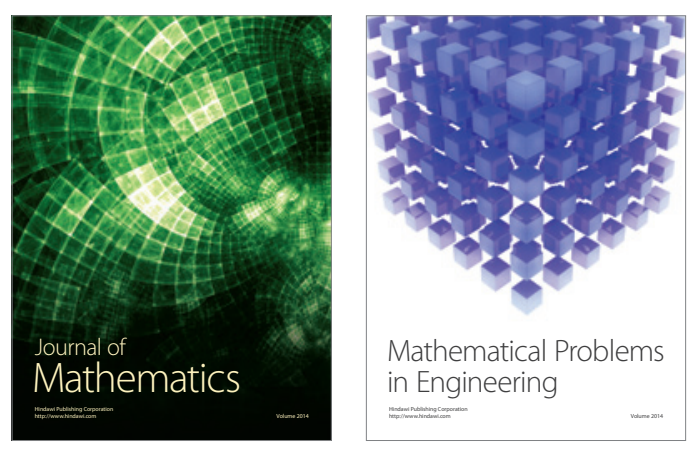

Mathematical Problems in Engineering
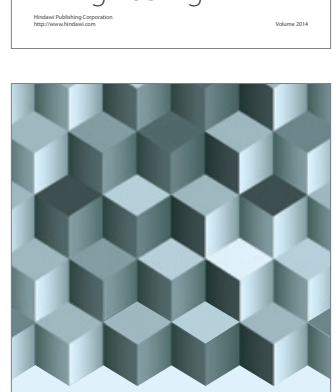

Journal of

Function Spaces
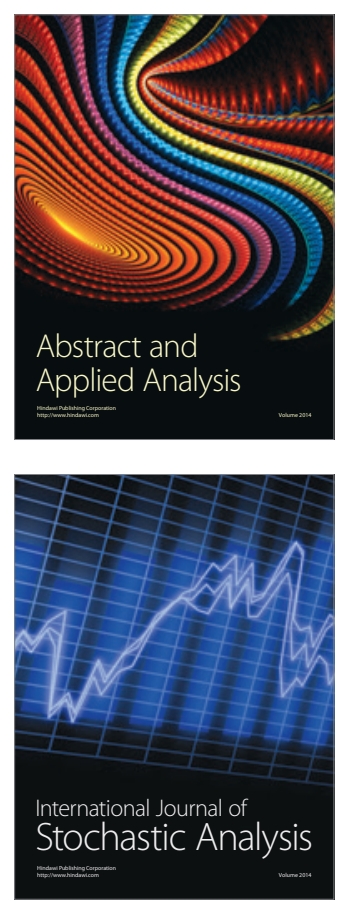

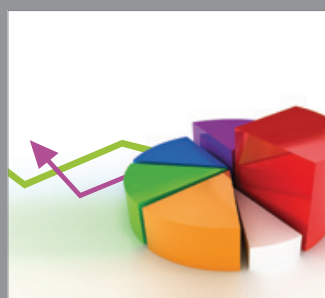

ournal of

Probability and Statistics

Promensencen
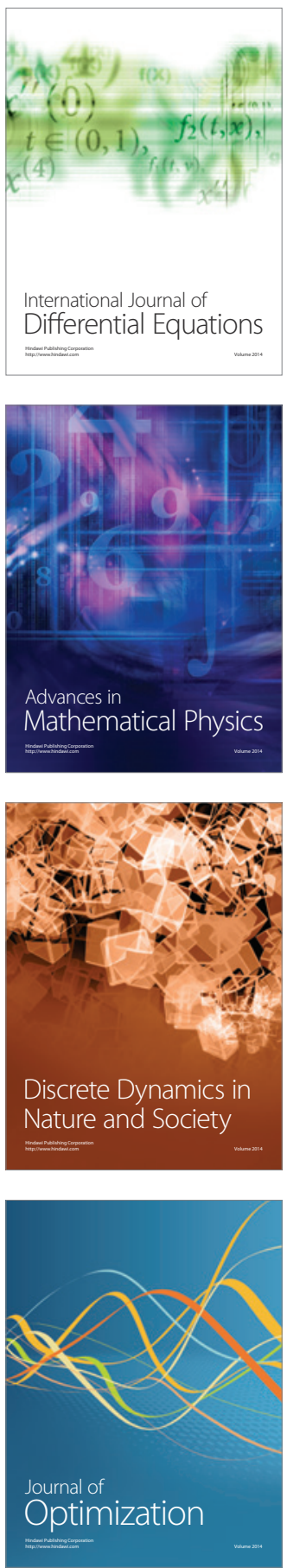